\title{
The three threats of action research: a discussion of methodological antidotes in the context of an information systems study
}

\author{
Ned Kock \\ College of Business and Economics, Lehigh University, 621 Taylor Street, Room 372, Bethlehem, PA 18015, USA
}

Received 1 July 2002; accepted 1 December 2002

Available online 10 March 2003

\begin{abstract}
This paper provides a brief historical discussion of action research (AR), from its emergence as a distinct research approach after World War II to its relatively recent use in the field of information systems (IS). Based on a review of the research methods literature, it presents and discusses three main threats inherent in action research, called "uncontrollability", "contingency", and "subjectivity"; and three methodological antidotes to deal with these three action research threats, called "unit of analysis", "grounded theory", and "multiple iterations". Both the threats and the antidotes are discussed in the context of a real information systems action research study that investigated the impact of computer support on the success of group-based business process improvement (BPI) attempts.
\end{abstract}

(C) 2003 Elsevier B.V. All rights reserved.

Keywords: Action research; Grounded theory; Information systems; Computer-mediated communications; Email; Business process improvement

\section{Introduction}

The emergence of organizational action research (AR) and its later use in the information systems (IS) field $[8,13]$ has been motivated by the recognition that an organization can be more deeply understood if the researcher is part of it, which can be achieved by the researcher facilitating improvement-oriented change in the organization $[6,17,24,25,39,62,73]$. This type of involvement is also believed to foster cooperation between researcher and those who are being studied, information exchange, and commitment towards both

E-mail address: nfk2@lehigh.edu (N. Kock). generating valid research conclusions and desirable organizational changes $[41,62,65,69]$.

Conducting organizational AR involves helping an organization solve its problems and become "better" in terms of some of its key attributes such as productivity, the quality of their products and/or services, and working conditions. At the same time, AR involves collecting, analyzing, and drawing conceptual and theoretical conclusions from organizational research data. This combination of "action" and "research" in organizational settings is perhaps the most appealing aspect of organizational AR $[25,93,94]$. In spite of the advantages that this combination of "action" and "research" can bring about, the use of AR in organizational research and, more specifically, in IS 
research, has been very limited $[69,71,83]$. This is surprising, particularly given AR's potential for generating outcomes that are relevant to industry practitioners pointed out by Truex [101] and highlighted by the guest-editors of a recently published special issue on IS AR of the journal Information Technology and People [65].

Obviously, there must be reasons why AR is underrepresented. A review of the research literature suggests that AR poses unique "threats" to research success $[46,47,79,87]$, which can potentially lead to a high proportion of failures in the conduct of AR and scare away potential adopters of AR as an approach for organizational research. This paper addresses this problem by presenting and discussing three fundamental "threats" posed by AR to researchers, as well as three methodological "antidotes" for the threats. Both the threats and the antidotes are discussed in the context of a real IS AR study that investigated the impact of computer support on the success of groupbased business process improvement (BPI) attempts [64]. The paper is organized as follows.

The section "Action research and its use in information systems" provides a brief historical discussion of $\mathrm{AR}$, from its emergence as a distinct research approach after World War II to its relatively recent use in the field of IS. This section also contrasts AR with other research approaches in IS. This is followed by the section "The three threats of action research", which presents and discusses three key threats posed by AR to researchers: Uncontrollability, contingency, and subjectivity. The discussion is based on a review of both the AR literature as well as the more general literature on research methods.

The section "Dealing with the action research threats: a discussion of three methodological antidotes" presents and discusses three methodological antidotes to deal with the AR threats identified in the previous section. The antidotes are developed from the general literature on research methods and are called unit of analysis, grounded theory, and multiple iterations. The following section, "Spotting the threats: a look at a real information systems action research study", discusses the three threats based on a real IS AR study whose main goal was to investigate the impact of electronic communication support on BPI groups. The following section, "Applying the methodological antidotes", discusses the application of the antidotes in the context of the IS AR study presented in the previous section. Finally, the "Conclusion" section concludes with a call for unity among those who subscribe to and practice AR and those who do not for reasons related to their epistemological orientation.

\section{Action research and its use in information systems}

Although there is controversy about its origins, AR seems to have been independently pioneered in the US and Great Britain in the early 1940s. Kurt Lewin is generally regarded as one of its pioneers $[6,25]$ through his work on group dynamics in the US. He is also believed to have been the first person to use the term "action research" [73]. Lewin [72] defined AR as a specific research approach in which the researcher generates new social knowledge about a social system, while at the same time attempting to change it $[21,72,85]$. A distinctive thrust of AR has also developed after World War II in Great Britain at the Tavistock Institute of Human Relations in London. There, AR was used as an innovative method to deal with sociological and psychological disorders arising from prison camps and war battlefields [41,87].

In AR, "action" and "research" are combined into a structured process usually referred to as the AR cycle [34], of which variations exist [76]. Perhaps the most widely accepted view of the AR cycle is that provided by Susman and Evered [97] in what is believed to be a seminal article that laid the foundations of modern organizational AR. Fig. 1 shows Susman and Evered's [97] AR cycle, which comprises

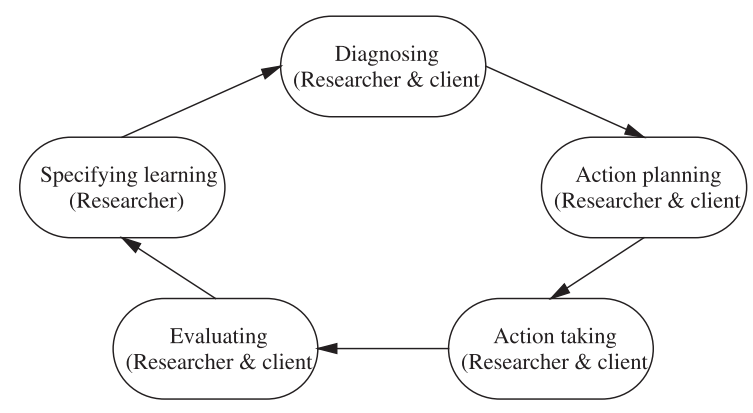

Fig. 1. Susman and Evered's [97] AR cycle. 
Table 1

Contrasting AR with other more traditional IS research approaches

\section{Experimental research}

Has its roots in the scientific practice of biologists, physicists, and physicians, where variables are manipulated over time, associated numeric data is collected, and causal or correlation models are tested through standardized statistical analysis procedures. The researcher has a strong control over the environment being observed.

Experimental research is typically applied to test models or hypotheses. Typical instances are Chidambaram and Jones' [26] study of the impact of communication medium and computer support on teams in dispersed and face-to-face meetings by comparing experiments where some groups used a group decision support system and others did not; and Gallupe et al.'s [49] comparative study where production blocking was manipulated in three experiments so the performance of blocked and unblocked electronic brainstorming and verbal brainstorming groups could be assessed.

Survey research

Has its roots in the work of economists and sociologists. The researcher typically has a considerable sample to be analyzed, which suggests the use of questionnaires with questions that are easy to be answered and that permit quantitative evaluation "a posteriori". Survey research is typically applied to test models or hypotheses. Typical instances are the survey involving 49 organizations in Southern California performed by Winter [102], which shows that computers can act as symbols of status; and Brynjolfsson and Hitt's [16] survey based on firm-level data from 1987 to 1991 about 380 large firms, which evaluates those firms' return on investment in IS.

\section{Case research}

Has its roots in general business studies, particularly those using what is referred to as the "Harvard Method" [40]. The researcher typically studies a small sample of organizations in depth. Cases are analyzed either to build up or validate models or theories, typically through collection of textual data in interviews. Typical instances are the on-site case research performed at a large corporation by Alavi [2] where she assessed the utilization of an electronic meeting system by employees; and the interview-based research performed by Trauth and O'Connor [100] to analyze the influences and impacts of cultural, economic, and political factors on the establishment and evolution of information technology firms in the Republic of Ireland.

\section{Action research}

Has its roots in studies of social and workplace issues. The researcher typically studies a small sample of organizations in depth, using participant observation and interviews as key data collection approaches. AR is often uniquely identified by its dual goal of both improving the organization participating in the research project, usually referred to as "client organization", and at the same time generating academic knowledge. Although typically applying very little, if any, control on the environment being studied, the researcher is expected to apply some form of "positive" intervention - typically as a service to the client organization. A typical instance is the research on the participatory development and introduction of an expert system in a welding plant conducted by Candlin and Wright [19]. five stages: Diagnosing, action planning, action taking, evaluating, and specifying learning. The diagnosing stage involves the identification by the researcher of an improvement opportunity at a prospective client organization that is likely to lead to the development of relevant knowledge. Action planning involves the joint development and consideration of alternative courses of action to attain the improvement identified and knowledge development. Action taking involves the selection and implementation of one of the courses of action considered in the previous stage. Evaluating involves the study of the outcomes of the selected course of action. Finally, specifying learning involves assessing the outcomes of the evaluating stage and, based on this assessment, knowledge generation in the form of a conceptual or theoretical model describing the situation under study.

While AR has been used in IS research since the 1980s [12], independent surveys conducted during the early and late 1990s lead to the conclusion that AR's representation in the field of IS has been very small $[69,83]$. Given AR's potential advantages for the conduct of IS research, particularly the likelihood that AR studies incorporate outcomes that are relevant to industry practitioners $[65,101]$ since AR often begins with the identification of problems faced by industry practitioners, the small representation of published AR studies in the field of IS comes as a surprise. The literature on IS research methods suggests that three approaches have accounted for most of the published investigations in the field: experimental, survey, and case research $[22,47,83]$. Table 1 , which builds on Kock et al.'s [67] discussion, provides a brief description of these three IS research approaches and contrasts them with AR.

The lack of AR representation in the IS literature becomes more understandable when some of AR's potential "threats" to research success and publication are investigated. This investigation is conducted next, with an emphasis on three threats that have been widely recognized not only by detractors of AR, but also by those who subscribe to and practice AR.

\section{The three threats of action research}

An investigation of the research methods literature, and in particular of the AR literature, suggests 
the existence of AR threats that have been repeatedly reported and that seem to require particular attention in the development of methodological tools for improving $\mathrm{AR}$ in general and ensure that its use in the field of IS will lead to successful research outcomes $[8,9,34,39,44-48,55,59,60,67,70,71,77,79,88$, $89,97]$. These threats, which are referred to here as uncontrollability, contingency, and subjectivity threats, seem to be associated with the "emergent" nature of most AR investigations [46,47], where a theoretical model emerges from the research data rather than being defined a priori and tested against that data [52]. This epistemological characteristic, present in the vast majority of AR investigations, and its associated problems, have also been addressed by Orlikowski and Baroudi's [83] methodological and epistemological discussion of IS research, and by Phillips and Pugh [86] in their comparative study of doctoral programs and approaches. Orlikowski and Baroudi's [83] widely cited article identifies similar threats as associated with research that does not conform closely with positivist research traditions, particularly research where the researcher interacts with the environment and the subjects being studied, and where theoretical models emerge from the research data rather than being tested against that data.

The focus of this paper on the uncontrollability, contingency, and subjectivity threats is motivated by the repeated references to instances of these threats and their negative research consequences in the AR literature, and their discussion in the general research literature in reference to research methods and approaches that have some of the key characteristics of AR. This suggests that these three threats require particular attention in the development of methodological guidelines for improving the outcomes of AR. Each of these threats is individually discussed below.

\subsection{The uncontrollability threat}

Even though in AR the researcher attempts to change the environment being studied, he or she does not usually have full control over that environment $[8,34,55,59,67]$. While this characteristic of AR facilitates the emergence of theoretical models from the research data $[52,97]$, it also creates problems for the researcher. The uncontrollability threat of AR comes from the fact that the researcher's degree of control over the environment being studied and the research subjects is always incomplete, even less so when the relationship between researcher and subjects begins with the AR study and has no prior history. In this respect, Avison et al. [9, p. 30] correctly point out that: "Rarely will an organization cede ultimate authority for organizational action to an external researcher. This guarded commitment is reasonable since the researcher's motives are divided between research goals and organizational problem-solving goals."

The essence of the uncontrollability threat is that while the environment being studied will often change in ways that have been predicted by the researcher, sometimes change will happen in ways that are completely unexpected [79]. The change may in some cases force the researcher to revisit his or her methods, theoretical assumptions, and even his or her research topic before a single iteration of the AR cycle is completed. Also, the researcher may be forced to abandon the research site before the study is completed due to events that are outside of his or her sphere of control $[67,79]$.

\subsection{The contingency threat}

In addition to not usually having full control over the environment being studied or study subjects, playing the role of an agent of change usually grants the researcher access to a considerably large body of data $[77,87,88]$, often more than he or she can handle $[66,97]$. The problem is that this body of data is usually "broad and shallow", rather than "narrow and deep" like the bodies of data collected through, for example, experimental research $[59,60,67,68]$. The vast body of "shallow" data collected through AR studies seldom provides cumulative evidence that points to a particular effect or refers to a particular construct, and is often difficult to analyze because the rich context in which it is collected makes it difficult to separate out different components that refer to particular effects or constructs [67,70,71,87].

The contingency threat comes from AR's inherent obstacles to isolation of evidence related to particular effects and constructs from the contextual "glue" in which they are naturally found. "Contingency" here is used as synonymous with difficulty to generalize research findings, or difficulty to apply the research 
findings in contexts different from the one in which they were generated. That is, highly contingent findings carry little external validity [15,29]. Regarding the degree of difficulty associated with the isolation and analysis of "units" of research data, AR could be seen as being at the high end of a scale of difficulty of effect and construct isolation, whereas experimental research could be seen as being at the low end of the scale $[39,67,88,89]$. In experimental research, the effect of one particular variable on another can be easily isolated through experimental controls. This is not possible in AR without making it lose some of the elements that characterize it as such-i.e., given AR's own definition, if an AR project employs control groups it can no longer be called AR, and should be seen as a field experiment $[46,48,57,67,88,89]$.

\subsection{The subjectivity threat}

The deep involvement of researchers with client organizations in AR studies may hinder good research by introducing personal biases in the conclusions [42]. This is particularly true in situations involving a conflict of interests. With respect to this, Galliers [46, p. 152] points out that AR “... places a considerable responsibility on the researcher when objectives are at odds with other groupings." While deep personal involvement from the part of the researcher has the potential to bias research results, it is inherent in AR because it is impossible for a researcher to both be in a detached position and at the same time exert positive intervention on the environment and subjects being studied. This is particularly true when the number of situations experienced by the researcher is small and the emotional intensity of this involvement is high. Research on human cognition has shown not only that human beings rely mostly on experiential learning for the acquisition of knowledge, but also that those experiences that are accompanied by intense emotional discharges (e.g., anger, fear) are remembered more vividly than those in which there is little emotion involved [50,91]. The downside of this phenomenon is that it is also likely to distort the way in which people in general, and AR practitioners in particular, may perceive events and situations where there is a high degree of personal involvement, especially when these situations involve conflict, stress, or any events that may lead to an emotional response.

The subjectivity threat hinges on the fact that, in $\mathrm{AR}$, the personal involvement of the researcher is likely to push him or her into interpreting the research data in particular and potentially subjective ways, and that, as a result, some of these interpretations may end up being completely wrong. Some common interpretation biases have been identified by the literature $[4,5,37,103]$, one of which is particularly relevant in the context of AR. That is the "externalization" bias, whereby an individual has difficulty assigning blame for "negative" outcomes of his or her own actions (e.g., dissatisfaction or frustration by his or her peers) to himself, instead trying to find ways to explain those "negative" outcomes based on factors that are external to him or her $[3,5,37]$. In IS AR, for example, this could lead to the wrong interpretation that certain "negative" behavioral patterns associated with dissatisfaction or frustration observed in research subjects are reactions to an information technology when in reality those behavioral patterns may be primarily motivated by the researcher himself and his or her own actions.

\section{Dealing with the action research threats: a discussion of three methodological antidotes}

In this section, principles are developed to address the three AR threats reviewed above. These principles are referred to as methodological "antidotes". Three methodological antidotes are proposed and individually discussed below. They are referred to as unit of analysis, grounded theory, and multiple iterations antidotes, and are based on three main methodological "tools". The first methodological tool is the unit of analysis method [30,38,104-106]. The second methodological tool is Glaser and Strauss's [53] grounded theory methodology (see also Refs. [51,52,95,96]). The third methodological tool is the multiple AR cycle iteration method $[61,67,97]$.

\subsection{The unit of analysis antidote}

The unit of analysis antidote is based on the use of the unit of analysis method [30,38,104-106], which prescribes that research data collection and analysis 
should be centered on units of analysis identified prior to the beginning of the research study. For example, units of analysis in IS AR research may be the "individual" user of information technology, or the "group" engaged in the use of a collaboration technology.

Usually, the more instances of a unit of analysis are studied in different contexts, the higher the external validity of findings relating patterns that are observed in different instances of the unit of analysis. Moreover, the more instances of a unit of analysis for which research data can be obtained, the more likely it is that statistical analysis techniques can be used to ascertain whether an observed trend (e.g., a particular behavior observed in many instances of the unit of analysis) is or is not due to chance $[30,38,54]$. And, ascertaining whether an observed trend is or is not due to chance is very important when the frequency distribution of the observed trend is scattered (i.e., the trend is not observed in all instances), which is often the case in AR [94].

The unit of analysis antidote counteracts the negative effects of all three AR threats when used in combination with the other methodological antidotes (this is discussed in the following sections), and particularly of the contingency threat, as it provides the basis on which to increase the external validity of research findings that refer to observable patterns in different instances of one or more units of analysis $[15,29]$. While this may seem obvious, the unit of analysis method has seldom been explicitly used in AR $[57,67,71]$.

\subsection{The grounded theory antidote}

The grounded theory antidote is based on the use of an adaptation of Glaser and Strauss's [53] grounded theory methodology. At the core of the grounded theory methodology is a three-step coding process, which is conducted in an iterative fashion and is aimed at increasing the reliability [20] of the analysis of large bodies of unstructured research data. That is, the coding process tries to foster objective data analysis and ensures that different coders, regardless of their level of involvement with the environment and subjects being studied, will produce the same (or similar) final data analysis results. The first step, open coding, involves the identification of emerging cate- gories in textual data. The second step, axial coding, involves the identification of relationships between the categories identified through open coding. The third and final step, selective coding, involves the grouping of interrelated categories into theoretical models $[51-53,95,96]$. The adaptation of this threestep coding process is necessary here because it has been originally proposed in a very generic format to allow for its adaptation to specific research needs and goals $[52,95,96]$.

In the adapted version proposed here for the specific needs of AR, grounded theory is used for the summarization of findings into causal models $[10,33]$ linking independent, moderating, intervening, and dependent variables derived from the study $[7,11,30,38]$. In addition, in the adapted version proposed here, open coding involves the identification of $n e w$ variables in any of the stages of the AR cycle, in addition to the variables related to units of analysis identified before the AR study begins based on theory [14]. Each variable is defined as an attribute of a unit of analysis that can vary on a numeric or non-numeric scale $[38,54,84]$. For example, the variable "cognitive effort", which may be associated with the use of a particular information technology, is an attribute of the unit of analysis "individual" that can vary along an ordinal scale containing the values "high", "medium" and "low". Similarly, the variable "cost" may be associated to a group's use of a particular collaboration technology, is an attribute of the unit of analysis "group", which can vary along a numeric scale. While open coding is used for the identification of additional variables (in addition to the ones identified prior to the beginning of the research study), axial coding becomes then the identification of links between variables, and selective coding becomes the identification of dependent variables, which act as "anchor" variables to which a set of interrelated variables and effects is associated. This process relies heavily on data tabulation [78] and to some extent on statistical analysis techniques (e.g., Chi-Square analysis), which will be illustrated later in this paper through the description of a real IS AR study.

Neither the intermediate steps taken in grounded theory development, nor the causal models generated through them necessarily have to be included in all reports, articles or papers on the AR study. The causal models generated are the highest level of abstraction 
regarding the findings of the study and serve as both a high-level representation of the main findings of the study, and an "index" against which different pieces of the intermediate research data can be found [52]. Since the coding of grounded theory is "directional", in that it takes the researcher from raw data to summarized data (often in tables) and finally to causal models, it becomes easy for an organized researcher (i.e., one who keeps organized records of the intermediate coding stages) to go back to the intermediate data that led to a particular causal link identified through selective coding $[95,96]$. Those intermediate data can then be shown as supporting evidence in a publication that focuses on a particular aspect of the AR study. Grounded theory development is a laborious process that is often "hidden" in the background and not explicitly referred to in publications beyond their "research method" sections $[53,95,96]$.

The grounded theory antidote counteracts the negative effects of all three AR threats when used in combination with the other methodological antidotes, and particularly those associated with the subjectivity threat, as it provides the basis on which to remove the subjectivity of the analysis of large bodies of data by fostering inter-coder reliability [20]. While grounded theory methodology has been extensively used in qualitative research in general $[95,96]$, explicit examples of its use in AR are difficult to find. For example, none of the papers in the two 1993 special Human Relations issues on AR explicitly employed it [39], nor did any of the papers in the 2001 special Information Technology and People issue on IS AR [65].

\subsection{The multiple iterations antidote}

The multiple iterations antidote is based on the conduct of multiple iterations of Susman and Evered's [97] AR cycle. One of the reasons for conducting multiple iterations of the AR cycle is the opportunity that it allows for collecting cumulative research data about specific units of analysis in different contexts and thus strengthening research findings by building on evidence gathered from previous iterations in the AR cycle. Ketchum and Trist [61] see the frequency of the iterations in the AR cycle as likely to decrease as the match improves between the researcher's con- ception of the phenomenon being studied, expressed in the causal models comprising the research findings, and that found as a result of the specifying learning stage in each subsequent AR cycle.

Multiple iterations of the AR cycle should expand the research scope, e.g., the areas of the client organization involved in the research, and build up the generality of the results through the identification of invariable patterns. The observation of invariable patterns in different contexts is a precondition for claiming external validity of research findings $[15,20,29]$. The point that multiple iterations of the AR cycle have the potential to expand the research scope and generality of the results is illustrated in Fig. 2, which depicts the relationship between research scope and the generality of the causal model describing research findings. The rectangles in the cycles represent each of the AR cycle stages, where: "di" represents diagnosis, "ap" represents action planning, "at" represents action taking, "ev" represents evaluating, and "sl" represents specifying learning. The iterations are named cycle 1 , cycle $2, \ldots$ to cycle $n$, where $n$ is the total number of iterations in the AR project.

The multiple iterations antidote counteracts the negative effects of all three AR threats when used in combination with the other methodological antidotes, and particularly those associated with the contingency and uncontrollability threats. Multiple iterations counter the negative effects of the contingency threat by allowing for the observation of invariable patterns in different contexts, for which a degree of external validity can be claimed $[15,20,29]$. The contribution of the multiple iterations antidote to counteract the uncontrollability threat comes from the lower reliance of the research on single iterations that it fosters, and the higher likelihood of success in subsequent iterations enabled by the cumulative experience gained by the researcher as the AR study progresses. In a single iteration study (i.e., one in which a single iteration of the AR cycle is conducted), problems can occur that are outside the sphere of control of the researcher and that can undermine the data collection and analysis. For example, with multiple iterations being conducted, the early termination of an iteration will not have as harmful an effect as if the entire AR study had been conducted as a single iteration study. Moreover, in a multi-iteration study, the experience gained in previous iterations helps the 


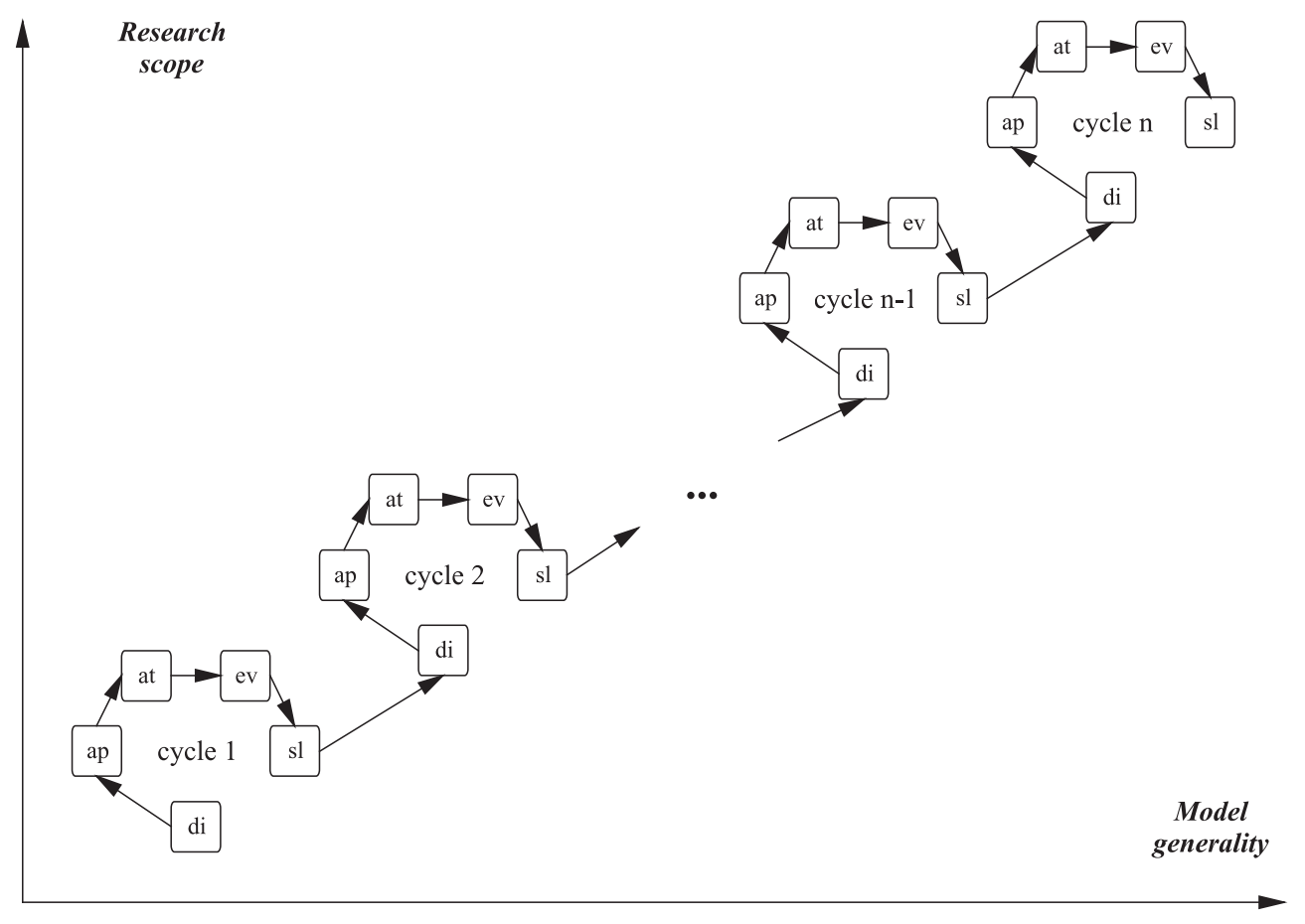

Fig. 2. Relationship between research scope and model generality (adapted from Ref. [67]).

researcher avoid situations that may jeopardize the $\mathrm{AR}$ study and come across to the client as more knowledgeable about the topic being studied and thus more worthy of the client's trust. While it seems that multiple iterations of Susman and Evered's [97] AR cycle are advisable, explicit examples of AR conducted through multiple iterations of the AR cycle are difficult to come by, as most AR studies seem to traverse the AR cycle only once $[39,65]$.

\section{Spotting the threats: a look at a real information systems action research study}

In this section, a real IS AR study is used to illustrate the occurrence of the three threats, where the author was the principal investigator. The use of the antidotes is discussed in the following section. The study was conducted through four iterations of Susman and Evered's [97] AR cycle that lasted approximately 4 years and 4 months. One of the iterations, the first, was conducted in Brazil. The other iterations were conducted in New Zealand. The focus of the study was on business process improvement (BPI) groups supported by Internet-based email conferencing systems, particularly regarding the impact of the technology on group success. The results of the study are discussed in more detail elsewhere $[63,64]$; the focus here is on the process of conducting AR from the perspective of the three threats and antidotes presented in the previous sections.

The first iteration of the AR cycle involved EventsInc (this and the other organizations are referred to by pseudonyms in this paper), a service firm in Brazil whose revenues came chiefly from the organization of large professional and trade events (e.g., exhibitions and conferences). The second and fourth iterations of the AR cycle were conducted at CollegeOrg, a comprehensive university in New Zealand. The third iteration of the AR cycle was conducted at GovernOrg, a branch of the Ministry of Agriculture and Fisheries of New Zealand.

Fig. 3 shows the main chronological stages of the study along a 4-year time scale that represents the full duration of the study. The iterations are briefly described at the bottom of Fig. 3. The top part of 


Contingency threat
$\cdot 1^{\text {st }}$ iteration of the AR cycle, specifying
learning stage.
-The lack of systematic data collection
about a single unit of analysis prevented
external validity claims.

\begin{tabular}{l}
\hline Uncontrollability threat \\
$\cdot 1^{\text {st }}$ iteration of the AR cycle, action \\
taking stage. \\
-Change in company ownership brought \\
the AR iteration abruptly to an end.
\end{tabular}

Subjectivity threat
$\cdot 3^{\text {st }}$ iteration of the AR cycle, specifying
learning stage.
- Conflict with a senior manager clouded
the researcher's perceptions.

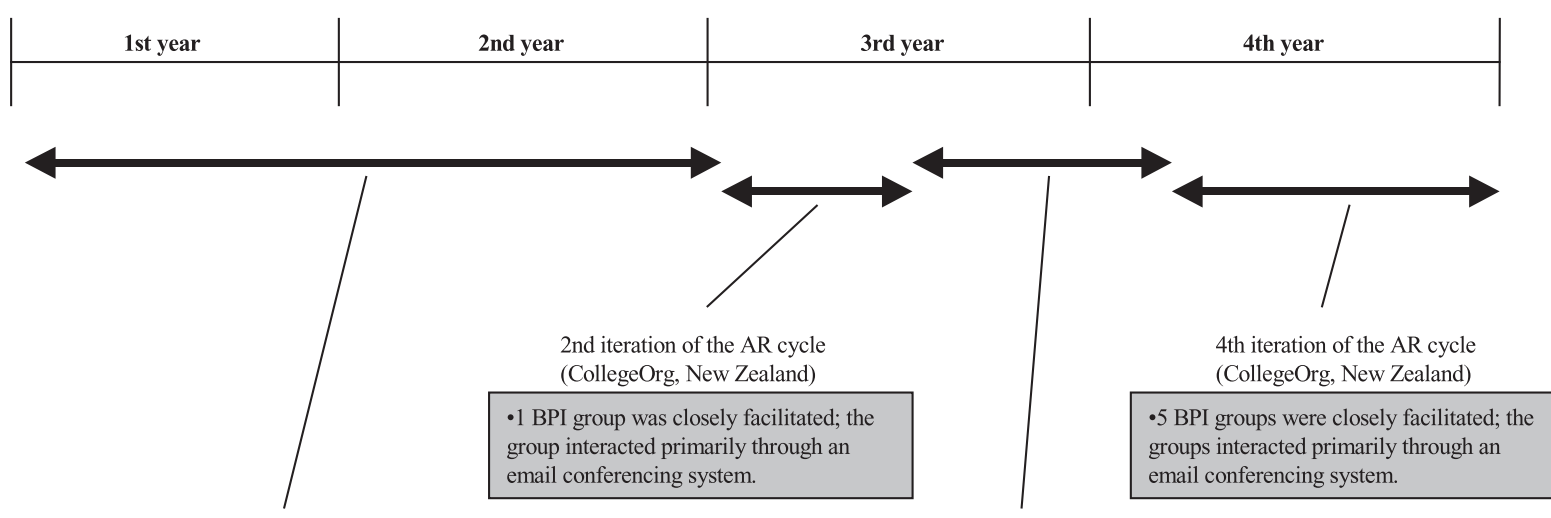

1st iteration of the AR cycle (EventsInc, Brazil)

-26 BPI groups were facilitated; 11 interacted only face-to-face; the other 15 used an email conferencing system.

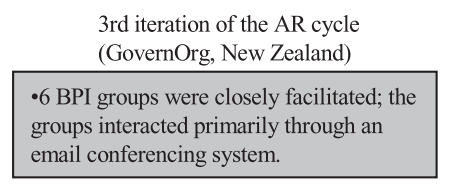

Fig. 3. Chronological view of the IS AR study.

Fig. 3 briefly describes instances of each of the three threats at their relative location on the time scale. The following subsections describe the motivation and theory behind the study, the structure of the BPI groups, data collection and analysis, and the different iterations of the study. A discussion of the threats is provided within sidebars at the points they emerged in the different iterations of the AR cycle (also indicated in Fig. 3).

\subsection{Motivation and theoretical background}

The research topic emerged from the researcher's work as a consultant, which involved facilitating business process improvement (BPI) groups in a variety of companies and helping them implement new improved business processes with information technologies. Most of the BPI groups facilitated involved members from different departments who discussed and tried to solve problems related to a business process whose component activities they had to routinely perform as part of their job.

In many organizations, participation in face-to-face BPI groups is disruptive for their members, since very often those members are not co-located. Given this, it seemed that BPI groups could benefit from the use of computer-mediated communication systems that allowed their members to interact in a distributed and asynchronous manner. When this study began, several technologies incorporated support for distributed and asynchronous electronic communication. One such technology was Internet-based email confer- 
encing (IEC). IEC seemed particularly appropriate to support BPI groups because it relied on an underlying technology, Internet email, which was simple and ubiquitous enough to enable seamless inter-organizational communication.

With some notable exceptions (see, e.g., Refs. [35, $36,74]$ ), most of the empirical evidence from the literature suggested grim expectations regarding the effect of IEC support on BPI groups, as electronic communication media have consistently been seen as less appropriate than the face-to-face medium to support the type of complex and knowledge-ridden communication that usually takes place in BPI groups [1,80-82,90,98].

The majority of the empirical evidence mentioned above was consistent with a few influential theories of organizational communication, such as social presence theory [92], and media richness theory [31]. These theories led to the conclusion that for group tasks as complex (or "equivocal", in media richness theory terminology) as BPI, asynchronous computer mediation would lead to outcomes that would be "worse" than those likely to be achieved by BPI groups interacting face-to-face [75]. On the other hand, another influential theoretical model, the social influence model $[43,75]$, argued that social influences could strongly shape individual behavior toward technology in ways that are relatively independent of technology traits. The social influence model suggested that certain social influences (e.g., perceived group mandate, peer expectations of individual behavior) could lead BPI members to adapt their use of technology in ways that were inconsistent with predictions based on the social presence and media richness theories, and achieve successful results. Therefore, a detailed investigation of IEC-supported BPI groups, focusing on the impact of IEC support on the success of the groups, seemed to combine practical relevance with the potential to contribute to a better understanding of these theoretical contradictions.

\subsection{Data collection and analysis}

The first iteration of the AR cycle provided anecdotal data about the potentially positive benefit of IEC support for BPI groups. This was confirmed based on a more focused data collection and analysis conducted in the second, third and fourth iterations of the $\mathrm{AR}$ cycle. In those iterations, perception frequency-based analyses of interviews triangulated with participant observation notes, electronic postings and other documents suggested that while BPI group members perceived IEC as a poor medium for BPI-related communication, IEC support caused a reduction in BPI group cost, an increase in the quality of the process redesign recommendations generated by BPI groups, and an increase in the rate of success of BPI groups. Fig. 4 summarizes aggregate research data obtained in the second, third and fourth iterations of the AR cycle regarding BPI group cost, outcome quality (i.e., quality of process redesign recommendations), and success.

The group cost and outcome quality summaries at the top of Fig. 4 refer to interview data and show the distribution of answers from BPI group members to questions in which they were asked about the impact of IEC support on group cost and outcome quality. The frequency distributions shown at the top of Fig. 4 were highly correlated with those of the BPI groups that succeeded (see bottom of Fig. 4), with Pearson correlation coefficients of 0.68 and above; the correlation coefficients were lower for the BPI groups that failed. Interviews were conducted with process improvement group members and addressed perceived differences between face-to-face and IEC groups they had participated in. To avoid perception bias, interview answers were probed deeply for rationale, personal motivations and other factors that could bias perceptions, as well as triangulated with other sources of data. Sixty-two structured and over one hundred unstructured interviews were conducted. The structured interviews, the core source of evidence in this study, were taped and later transcribed. They employed an "in-depth interviewing" method proposed by Sommer and Sommer [94], lasted from 45 min to $2.5 \mathrm{~h}$ each, and were based on open-ended questions. The open-ended questions used in structured interviews were worded in a neutral way so as not to induce any specific answer. Each question was accompanied by the follow up question "Why?" and other related questions to clarify the interviewees' motivations for their answers, allow for the screening and elimination of ambiguous answers, and generate perception-related qualitative data that could be used for content analysis. The frequency distributions of interview answers were tested for statistical signifi- 
Group cost

\begin{tabular}{lll}
\hline Answer & Percentage & Frequency \\
\hline Increased & $1.6 \%$ & 1 \\
Decreased & $88.7 \%$ & 55 \\
Had no effect & $4.8 \%$ & 3 \\
I don't know & $4.8 \%$ & 3 \\
\hline Chi-Square & $>100$ & \\
P & $<.01$ & \\
\hline Chi-square parameters: $\boldsymbol{N}=62$, df $=2$ \\
\multicolumn{1}{l}{ ("I don't know" answers were disregarded) }
\end{tabular}

Group outcome quality

\begin{tabular}{lll}
\hline Answer & Percentage & Frequency \\
\hline Increased & $43.5 \%$ & 27 \\
Decreased & $21.0 \%$ & 13 \\
Had no effect & $25.8 \%$ & 16 \\
I don't know & $9.7 \%$ & 6 \\
\hline Chi-Square & 5.84 & \\
P & $<.05$ & \\
Chi-square parameters: $\mathbf{N}=62$, df $=2$ & \\
\multicolumn{1}{l}{ ("I don't know" answers were disregarded) }
\end{tabular}

Group success

\begin{tabular}{|c|c|c|c|c|c|}
\hline Group & Successful? & Description & Group & Successful? & Description \\
\hline CollegeOrg.G1 & Yes & $\begin{array}{l}\text { Group agreed on process changes, from } \\
\text { which all were implemented with positive } \\
\text { results }\end{array}$ & GovernOrg.G1 & Yes & $\begin{array}{l}\text { Group agreed on process changes, from } \\
\text { which approximately two thirds were } \\
\text { implemented with positive results }\end{array}$ \\
\hline CollegeOrg.G2 & No & $\begin{array}{l}\text { Group agreed on process changes, from } \\
\text { which none was actually implemented }\end{array}$ & GovernOrg.G2 & Yes & $\begin{array}{l}\text { Group agreed on process changes, from } \\
\text { which all were implemented with positive } \\
\text { results }\end{array}$ \\
\hline CollegeOrg.G3 & No & $\begin{array}{l}\text { No agreement on process changes was } \\
\text { achieved }\end{array}$ & GovernOrg.G3 & Yes & $\begin{array}{l}\text { Group agreed on process changes, from } \\
\text { which approximately one third were } \\
\text { implemented with positive results }\end{array}$ \\
\hline CollegeOrg.G4 & Yes & $\begin{array}{l}\text { Group agreed on process changes, from } \\
\text { which all were implemented with positive } \\
\text { results }\end{array}$ & GovernOrg.G4 & No & $\begin{array}{l}\text { No agreement on process changes was } \\
\text { achieved }\end{array}$ \\
\hline CollegeOrg.G5 & Yes & $\begin{array}{l}\text { Group agreed on process changes, from } \\
\text { which all were partially or fully } \\
\text { implemented with positive results }\end{array}$ & GovernOrg.G5 & Yes & $\begin{array}{l}\text { Group agreed on process changes, from } \\
\text { which approximately three quarters were } \\
\text { implemented with positive results }\end{array}$ \\
\hline CollegeOrg.G6 & Yes & $\begin{array}{l}\text { Group agreed on process changes, from } \\
\text { which all were partially or fully } \\
\text { implemented with positive results }\end{array}$ & GovernOrg.G6 & No & $\begin{array}{l}\text { Unfocused discussion led the group to be } \\
\text { discontinued without agreeing on any } \\
\text { process changes }\end{array}$ \\
\hline
\end{tabular}

Success rate $=67 \%$ (over twice the $30 \%$ success rate obtained from the literature)

Fig. 4. Summary of the research data on group cost, outcome quality, and success.

cance using the Chi-Square technique. The ChiSquare tests excluded ambiguous and "I don't know" answers (both placed in the "I don't know" category when frequency distributions were calculated), which added robustness to the results. The frequency distributions of answers in interviews were similar for CollegeOrg and GovernOrg (Cronbach Alpha= 0.72).

The group success summary at the bottom of Fig. 4 refers to a categorization of groups as successful or not according to success criteria proposed by the BPI literature [18,32,56]. The group CollegeOrg.G1 was conducted at CollegeOrg in the second iteration of the AR cycle. The groups CollegeOrg.G2 to CollegeOrg.G6 are the five BPI groups that were conducted at CollegeOrg during the fourth iteration of the AR cycle. The groups GovernOrg.G1 to GovernOrg.G6 are the six BPI groups conducted at GovernOrg during third iteration of the AR cycle. These iterations are described in some detail in the following sections.

As it can be seen in Fig. 4, 88.7\% of the participants felt that IEC support decreased group cost. The perception trend toward a decrease in group cost due to IEC support was very skewed and statistically significant (Chi-Square $>100, P<0.01$ ). Most of the respondents described the reduction in cost as being "drastic", and as being caused by a virtual elimination of travel and accommodation costs, a reduction in the total amount of time required from them to participate in group discussions, and a reduction in the costs associated with disruption of normal activities normally caused by face-to-face meetings.

As shown in Fig. 4, group outcome quality, or the quality of the process redesign recommendations generated by BPI groups, was perceived by $43.5 \%$ of the participants as having been increased by IEC support. The perception trend toward an increase in group outcome quality was statistically significant $($ Chi-Square $=5.84, P<0.05)$. The two main reasons independently provided by BPI group members to explain why they thought IEC support had increased group outcome quality were a better quality of individual contributions and a higher departmental heterogeneity than in similar face-to-face process 
improvement initiatives, both seen as fostered by IEC support. On the other hand, $21 \%$ of the BPI group members perceived a decrease in quality associated with the IEC support. The main and virtually only reason provided to explain this perception was that the IEC medium was not as "good", "rich", or "appropriate" as the face-to-face medium for BPI group discussions, increasing the level of ambiguity in them. Compilations of electronic postings suggest that, in most cases, those who perceived a decrease in group outcome quality due to IEC support had not participated actively in the BPI group discussions, in some cases having completely withdrawn from the discussions right after their start. Also, most of those members were from BPI groups that failed.

The bottom part of Fig. 4 suggests that four out of the six process improvement groups conducted at CollegeOrg, as well as four out of the six BPI groups conducted at GovernOrg, were successful. The criteria for success used were derived from the BPI literatureBPI attempts are considered successful if the recommended process changes are implemented fully or partially and lead to positive observable results $[18,23,27,32,56]$. The results summarized in Fig. 4 indicate that, overall, 8 out of 12 groups were successful, which yields a total success rate of $67 \%$. This success rate is over twice the success rate of BPI attempts based on total quality management and business process re-engineering principles, which the literature suggests to be around $30 \%$ or less $[23,27]$. That is, the rate of success of the IEC-supported BPI groups studied was significantly higher than the average suggested by the literature.

In summary, the results above suggest a reduction in BPI group cost, an increase in BPI group outcome quality, and an increase in success rate of BPI groups, as associated with IEC support. The results partially support and, at the same time, contradict the social presence [92] and media richness [31] theories. While the evidence suggesting that IEC was consistently perceived as a poor medium for BPI-related communication partially supports the theories, the increase in the quality of the process redesign recommendations generated by the groups and in their success rate contradicts expectations based on the theories. Fulk et al.'s [43] social influence model provides a explanation for these contradictory results, by showing that social influences (e.g., perceived group mandate, peer expectations of individual behavior) could lead BPI members to adapt their use of technology in ways that are inconsistent with predictions based on the social presence and media richness theories, and achieve successful results by compensating for the obstacles posed by a medium of low social presence and richness. This is what seems to have happened, as indicated by one of the two key explanations provided by BPI group members who perceived an increase in group outcome quality, which was a perceived increase in the quality of individual contributions fostered by the IEC medium. Therefore, the combination of the social presence and media richness theories with the social influence model provides a solid basis on which to fully understand the results summarized above.

From an empirical perspective, the findings contradict most of the empirical literature on distributed group support systems $[1,80-82,90,98]$, and provide the basis on which to argue that rational choice $[31,92]$ and social theories [43] of organizational communication can be combined to understand the behavior of complex and knowledge-intensive groups in real organizational situations. Previously, these types of theories had often been pitted against each other and presented as incompatible.

Below, a description of each of the iterations of the AR cycle is provided. These iterations led to the results summarized above. Their description highlights the three threats of AR, which are discussed at the points they emerged within the iterations. A discussion of how the methodological antidotes were employed to deal with the threats is provided in the section following the sections describing the four iterations of the AR cycle below.

\subsection{The first iteration of action research cycle: EventsInc in Brazil}

\subsubsection{Diagnosing}

EventsInc's local area network of computers was not working properly, which prevented the full deployment of an email package they had purchased a while ago. Since the researcher was interested on the impact of IEC support on BPI groups, which required a working email system, and had some local area network installation and set up skills, EventsInc saw this as an opportunity to have their technical networking problems solved. 


\subsubsection{Action planning}

Client organization and researcher planned to conduct the iteration of the AR cycle over approximately 1 year, starting with the solution of the networking problems and then moving on to the running of IECsupported BPI groups.

\subsubsection{Action taking}

The computer networking problems were fixed and the email conferencing system was installed without any major problems, but a little later than originally planned. The system allowed BPI groups to post electronic messages onto mailboxes created for each group discussion. Twenty-six BPI groups were conducted. The first 11 of those BPI groups interacted only face-to-face because the email conferencing system was not yet available; the others conducted most of the discussions via the IEC system. Most of the groups generated BPI proposals in no more than 40 days, which were in most cases implemented immediately after the completion of the BPI groups.

At the end of the "action taking" stage in the first iteration of the AR cycle, a bizarre turn of events (described in the sidebar below) took place. This turn of events provides a good illustration of the uncontrollability threat.

\subsubsection{Evaluating}

Given the turn of events described above, there was urgency to analyze the data for the report to the acquiring company. Anecdotal evidence from interviews triangulated with participant observation notes, electronic postings and other documents suggested that the project had been very successful from an organization-wide perspective, even though its final outcome had been less than positive in the eyes of several of its key members. Significant efficiency gains in local processes due to the decentralization of access to information, a major simplification of the organization's departmental structure, and an increase in revenues were the main bottom-line results of the changes brought about by the BPI groups.

\subsubsection{Specifying learning}

Having just left the research site, the researcher found himself overwhelmed not only by the large body of data to be analyzed but also by important decisions that had to be made in order for him to be able to produce what he saw as "relevant knowledge". Producing such knowledge is the main goal of the "specifying learning" stage of the AR cycle [97].

At this point, it became clear that the researcher's broad and unfocused data collection had led him to

\section{The uncontrollability threat}

\section{The truth about EventInc's management's real intentions}

The researcher expected EventsInc's management to want competitors to be as far away as possible from the company's premises so as to prevent them from copying EventsInc's new approach to BPI. Nevertheless, in several occasions, the chief executive officer invited the owners of a competing company to see the intermediate results of the project. The visitors, who were introduced to the researcher as "some friends" by the chief executive officer, usually asked the researcher questions about the impact of BPI groups on EventsInc's bottom line (e.g., sustained increases in sales, profitability, etc.).

Approximately 9 months into the project, the researcher heard from one irate executive that EventsInc was undergoing the first stages of an amicable acquisition by a competitor, who turned out to be the one whose representatives had been visiting EventsInc and asking him questions. The AR iteration was discontinued and the researcher was asked to conduct an analysis of the project and summarize it in a report for the acquiring company.

As soon as the news about the acquisition became public, key employees left the company in disgust. Conversations with management and employees suggested that the general feeling was that the BPI project had been used to "add market value" to the company. 
fall prey to AR's contingency threat, which is discussed in the sidebar below. He had not been able to collect enough data to reach valid conclusions about the impact of technology on BPI groups, and the turn of events since the announcement of the acquisition prevented him from collecting any additional data.

\section{The contingency threat}

\section{The broad and unfocused data collection led to very context-specific lessons}

Every observable event, comment by a BPI group member, printed document, electronic posting, etc., had been a data point for the researcher, which led him to observe a number of effects that could have influenced BPI group success. The combination of this abundance of data with the company sell-out made him lose focus and consider research questions that had nothing to do with his initial theoretical motivations. For example, should the use of groupware-supported BPI by management as a means of (unethically) adding value to a soon-to-be-sold company be the main focus of his analysis, or should the target of his analysis be the impact of groupware on BPI groups?

The researcher eventually decided to use the data that he had at hand to try to reach some conclusions about the impact of IEC support at the group level of analysis. His unfocused data collection led him to reach conclusions that were very tentative and accompanied by several caveats and limitations. No unequivocal theoretical conclusions were possible.

\subsection{The second iteration of the action research cycle: CollegeOrg in New Zealand}

Soon after the first iteration was completed, the researcher migrated to New Zealand (something that he had planned to do, for personal reasons), and continued his research on IEC-supported BPI groups there. The second iteration of the AR cycle, carried out at CollegeOrg, was narrow is scope, and helped the researcher become familiar with the New Zealand culture as well as establish a solid basis for further iterations in that country.

\subsubsection{Diagnosing}

CollegeOrg had recently developed a hands-on introductory computing course covering a number of software applications, including email, group decision support systems, Internet Web browsers, word processors, spreadsheets and data base management systems. While attracting much interest from students, with about 100 enrolments per semester, the course had recently been the focus of many student complaints related to course design, level of work required, computer lab scheduling and other related issues. One of the instructors involved in teaching the course thought that an IEC-supported BPI group could help solve these problems in a more expedited fashion than a face-to-face group.

\subsubsection{Action planning}

The researcher quickly designed and implemented an IEC system similar to the one he had developed at EventsInc. The instructor mentioned above invited several individuals to participate in the BPI group. The instructor planned to address the course problems through an IEC-supported BPI group discussion lasting no more than 3 months so that the agreed upon changes in the process (i.e., the process of teaching the course) could be implemented in time for the following semester.

\subsubsection{Action taking}

The BPI group lasted 33 days and comprised seven members from a computer support area and one academic department. The interaction in the group comprised 21 postings, and a number of one-on-one phone and face-to-face conversations. According to estimates provided by group members, the vast majority of the time spent by group members in the group discussion was in interactions through the IEC system, with a small amount of time spent in oral one-on-one interactions. The group was completed in time for the 
implementation of the process changes to be assessed in the following semester. The impact of the process changes was assessed through a survey of student perceptions about the course. The survey covered most of the points targeted by the BPI group. It indicated a remarkable improvement in the quality of the course, when compared with a previous survey performed in the previous semester.

\subsubsection{Evaluating}

Perception frequency analyses of interviews triangulated with participant observation notes, electronic postings and other documents suggested that, while BPI group members perceived IEC as a poor medium for BPI-related communication, BPI group cost had been reduced (due to a reduction in the total amount of time required from members to participate in group discussions) and the quality of the process redesign recommendations generated by the group had been increased by IEC support. These effects, when combined, suggested that IEC support could increase the success of BPI groups, but it was clear that more evidence was needed to assess the validity of this conclusion.

\subsubsection{Specifying learning}

The results from the "evaluating" stage both supported and contradicted the social presence [92] and media richness [31] theories. The majority perception that IEC was a poor medium for BPI-related communication partially supported the theories. However, the apparent increase in the quality of the process redesign recommendations generated by the group contradicted the theories. As predicted, Fulk et al.'s [43] social influence model provided an explanation for these contradictory results.

\subsection{The third iteration of the action research cycle: GovernOrg in New Zealand}

The third iteration of the AR cycle involved circumstances close to ideal for the evaluation of the impact of IEC on BPI groups. Not only did GovernOrg conduct BPI groups before, primarily face-to-face and without the support of electronic communication tools, but it also presented a very geographically fragmented office distribution in New Zealand, which rendered it a prime client for an AR project involving IEC-supported BPI groups. However, this iteration also gave the researcher a "taste" of AR's subjectivity threat.

\subsubsection{Diagnosing}

GovernOrg's chief executive officer had recently issued a warning about the prospect of imminent deregulation of New Zealand's food production sector, whose government-mandated quality standards were inspected by GovernOrg, and the consequent privatization of GovernOrg, which would then become an independent auditing and consulting firm. GovernOrg's management viewed IEC-supported BPI groups as an opportunity to improve several of GovernOrg's processes in time so it could be prepared for the transition from a government branch to a private firm.

\subsubsection{Action planning}

The researcher quickly implemented an IEC system similar to the one that had been used at CollegeOrg in the previous iteration of the AR cycle, a task that was made easier by the fact that both CollegeOrg and GovernOrg used the same email server softwareNovell Groupwise (Novell Corp.). It was decided that the availability of the IEC system for the conduct of BPI groups, as well as the researcher's technical facilitation, would be announced to managers and employees, who would also be invited to voluntarily form and conduct BPI groups using the IEC system with the researcher's technical facilitation.

\subsubsection{Action taking}

Six BPI groups were conducted. The groups lasted from 10 to 29 days, had from 5 to 15 members, and altogether involved 47 managers and employees from 18 different office sites spread throughout New Zealand. Each office site typically supplied a number of services to customers in a town or city and vicinities. In most BPI groups, the process redesign recommendations were implemented with observable increases in process efficiency and/or solution of quality-related problems.

\subsubsection{Evaluating}

Perception frequency analyses of interviews triangulated with participant observation notes, electronic postings and other documents again suggested that BPI group members perceived IEC as a poor medium for BPI-related communication. Also, the data again 
suggested a reduction in BPI group cost and an increase in the quality of the process redesign recommendations generated by the groups. According to success criteria proposed by the BPI literature, the rate of success of the BPI groups conducted at GovernOrg had been approximately $67 \%$, which was over twice the approximately $30 \%$ success rate reported in the BPI literature for BPI groups in general. That is, the evidence in this iteration was very similar to that obtained in the previous iteration and pointed at the same technology effects.

At the beginning of the "specifying learning" stage in the third iteration of the AR cycle, a conflict erupted between a senior manager from GovernOrg and the researcher, which is described in the sidebar below. This conflict and its consequent biasing effect on the researcher's interpretation of part of the research data, provide a good illustration of AR's subjectivity threat.

\section{The subjectivity threat}

\section{A conflict with a senior manager biased the researcher's interpretation of the evidence}

At GovernOrg, two senior executives who reported directly to the chief executive officer had sanctioned the AR iteration to be conducted in their divisions. It became clear as the research progressed that these two senior executives had very different personalities and management styles. One adopted a very democratic and consultative management style, whereas the other adopted a much more autocratic style. Their perceptions of IEC-supported BPI groups were equally distinct. The democratic manager's view of IEC-supported BPI groups was very positive. The autocratic manager, on the other hand, felt that IEC-supported BPI groups were "a big waste of time", as well as an "obstacle" to swift senior management decisions.

The researcher had not been given an office at GovernOrg. Therefore, he usually conducted interviews in either the interviewee's office or the local cafeteria. In the middle of one of those interviews, at one of the tables in the cafeteria, the autocratic manager approached him and said, in a loud voice, that he had a "very cushy" lifestyle, since he (i.e., the researcher) was always "taking breaks" at the cafeteria.

The researcher was offended by the unfair public reprimand by the autocratic manager. His self-pity and pride led him to explain the incident based on the notion that electronic communication empowers employees [28] and that autocratic managers, like the one who had just accused him of having a "very cushy" lifestyle, do not know how to deal with that empowerment very well.

However, while coding data from an interview with the autocratic manager, conducted after the incident at the cafeteria, and comparing it with his participant observation notes, it became clear that one of the researcher's actions at the beginning of the research iteration had potentially had a much stronger impact than technology itself on how the autocratic manager perceived the researcher and anything that had to do with him, including the IEC-supported BPI groups. Early in the third iteration of the AR cycle, the researcher had conducted a simple quality and productivity audit at GovernOrg. That audit, which was conveyed to both managers, revealed that the productivity (assessed by standard metrics such as "revenues per employee") of the democratic manager's division was higher than that of the autocratic manager's division.

\subsubsection{Specifying learning}

As with the previous iteration of the AR cycle, the results of the "evaluating" stage in this iteration of the AR cycle supported and, at the same time, contradicted the social presence [92] and media richness [31] theories. Again, as in the previous iteration of the AR cycle, Fulk et al.'s [43] social influence model seemed to provide a complementary explanation for these contradictory results.
5.6. The fourth iteration of the action research cycle: CollegeOrg in New Zealand

The similarity between the findings of the second and third iterations of the AR cycle suggested, according to the "saturation" criterion proposed by Ketchum and Trist [61], that the AR study could be concluded after the third iteration. However, the researcher felt that he should go back to CollegeOrg 
and collect more BPI group data to ensure that the findings of the second iteration were not idiosyncratic. He did this in his fourth iteration of the AR cycle.

\subsubsection{Diagnosing}

After the second iteration of the AR cycle was concluded, CollegeOrg's president had launched an organization-wide initiative to enhance CollegeOrg's image as a top-quality tertiary education institution in New Zealand. A number of process efficiency and quality gaps were identified as a result of this initiative. Since the researcher had been discussing his AR study with several of CollegeOrg's faculty and staff while conducting the third iteration of the AR cycle at GovernOrg, many of them saw IEC-supported BPI groups as a way to deal with the productivity and quality gaps identified as a result of CollegeOrg's president's initiative.

\subsubsection{Action planning}

Several interested faculty and staff approached the researcher to provide technical facilitation for voluntary BPI groups using the IEC system employed at CollegeOrg in the second iteration of the AR cycle. It was decided that five BPI groups, whose membership and general problems to be addressed had been agreed upon in advance, would be conducted with the researcher's technical facilitation. This would also allow the researcher to collect data about the same number of groups, i.e., six, in CollegeOrg and GovernOrg, which provided some balance of data sources to his AR study.

\subsubsection{Action taking}

As planned, five BPI groups were conducted. The groups lasted from 32 to 54 days, had from 7 to 13 members, and altogether involved 48 faculty and staff from 15 different departments. In most BPI groups the process redesign recommendations were implemented with observable increases in process efficiency and/or solution of quality-related problems.

\subsubsection{Evaluating}

Perception frequency analyses of interviews triangulated with participant observation notes, electronic postings and other documents again suggested that BPI group members perceived IEC as a poor medium for
BPI-related communication. Also, the data again suggested a reduction in BPI group cost and an increase in the quality of the process redesign recommendations generated by the group. Again, according to success criteria proposed by the BPI literature, the rate of success of the BPI groups conducted at CollegeOrg was over twice the average reported in the BPI literature. That is, the evidence in this iteration was very similar to that obtained in the two previous iterations and pointed at the same technology effects.

\subsubsection{Specifying learning}

As with the two previous iterations of the AR cycle, the results of the "evaluating" stage in this iteration of the AR cycle supported and, at the same time, contradicted the social presence [92] and media richness [31] theories. Again, as in the two previous iterations of the AR cycle, Fulk et al.'s [43] social influence model provided a plausible explanation for these contradictory results.

\section{Applying the methodological antidotes}

As mentioned before, the combined use of the three methodological antidotes-unit of analysis, grounded theory, and multiple iterations - has the potential to counteract the negative effects of all three AR threatsuncontrollability, contingency and subjectivity. The unit of analysis antidote drives the cumulative collection and analysis of data about the same unit of analysis in different contexts, which counteracts the contingency threat by reducing the context-specificity of the research findings regarding units of analysis about which cumulative data was collected and analyzed. The grounded theory antidote entails the use of a reliable research data coding method that makes data analysis more objective, which counteracts the $s u b$ jectivity threat. The multiple iterations antidote entails the conduct of AR through multiple iterations of the AR cycle, which counteracts the uncontrollability threat by reducing the impact that events outside the sphere of control of the researcher, such as the early termination of one single iteration, have on the AR study as a whole. This is summarized in Table 2, which also describes specific instances in the AR study just reviewed that illustrate the contribution of the methodological antidotes to counteracting the threats. 
Table 2

Application of the methodological antidotes

\begin{tabular}{|c|c|c|c|}
\hline Threat & Uncontrollability & Contingency & Subjectivity \\
\hline Antidote & Multiple iterations & Unit of analysis & Grounded theory \\
\hline General result & $\begin{array}{l}\text { The decision to perform } \\
\text { multiple iterations of the } \\
\text { AR cycle reduced the } \\
\text { impact of events outside } \\
\text { the researcher's sphere of } \\
\text { control from negatively } \\
\text { affecting the AR study } \\
\text { as a whole. }\end{array}$ & $\begin{array}{l}\text { The study of different } \\
\text { instances of the same } \\
\text { unit of analysis, } \\
\text { BPI group, in different } \\
\text { contexts countered the } \\
\text { contingency threat. }\end{array}$ & $\begin{array}{l}\text { The grounded theory coding } \\
\text { process made the data analysis } \\
\text { more objective and less likely } \\
\text { to be contaminated by the } \\
\text { researcher's personal biases. }\end{array}$ \\
\hline Specific result & $\begin{array}{l}\text { Even though the first } \\
\text { iteration contributed } \\
\text { little to the final findings, } \\
\text { it helped the researcher } \\
\text { gain experience that he } \\
\text { used in subsequent iterations. }\end{array}$ & $\begin{array}{l}\text { The focus on the unit } \\
\text { of analysis "BPI group" } \\
\text { made the findings obtained } \\
\text { in and after the second } \\
\text { iteration less "contingent" } \\
\text { on context than those in } \\
\text { the first iteration. }\end{array}$ & $\begin{array}{l}\text { The systematic coding and } \\
\text { summarization of data } \\
\text { forced the researcher to } \\
\text { review his initial explanation } \\
\text { for the autocratic manager's } \\
\text { hostility in the third iteration. }\end{array}$ \\
\hline
\end{tabular}

The researcher's decision to conduct the study through multiple iterations of the AR cycle, which is the essence of the multiple iterations antidote, led him to use the experience obtained in previous iterations to avoid problems that characterize the uncontrollability threat of AR. For example, it led him to turn down an invitation from a chief executive officer of a New Zealand company who seemed to view the AR study as an opportunity to impose a BPI program on his employees in order to obtain a quality certification; an invitation that took place in between the first and the second iterations of the AR cycle. Moreover, the use of multiple iterations prevented the early, and relatively "traumatic", termination of the first iteration of the AR cycle from compromising the AR study as a whole.

The researcher's decision to focus his data collection and analysis on a specific unit of analysis, which is the essence of the unit of analysis antidote, in and after the second iteration of the AR cycle, led him to generate findings with potentially high external validity $[15,20,29]$, and thus avoid the contingency threat. It did so by allowing the researcher to observe patterns related to the same unit of analysis, the "BPI group", which repeated themselves in different contexts. Among these patterns were a reduction in group cost, an increase in group outcome quality, and an increase in group success, in connection with the use of the IEC system by group members.
Finally, the researcher's decision to employ the three-stage coding process prescribed by Glaser and Strauss's [53] grounded theory methodology, which is the essence of the grounded theory antidote, in and after the second iteration of the AR cycle, helped him counter the subjectivity threat by allowing the researcher to analyze the research data in a more objective way, thus preventing his personal biases from clouding his research conclusions. The systematic coding and summarization of data that characterizes grounded theory methodology led the researcher to evidence that forced him to review his technology-based explanation for the autocratic manager's hostility toward him at the end of the third iteration of the AR cycle, and consider the alternative explanation that his own previous actions had triggered that hostility.

\section{Conclusion}

The discussion of threats and antidotes, conducted in this paper, illustrates an area of methodological inquiry that has met with some resistance in certain AR circles in the past $[57,88,89]$, but that can potentially lead to highly desirable outcomes, which is the adaptation and use of established research methods and techniques in the context of AR. The resistance has been motivated by the assumption held in some 
AR circles that AR is somehow opposed to positivism, and thus should not adhere to positivist research methods and techniques [67,89]. This creates problems, since a large number of very useful research methods, techniques, and notions, have been developed in the context of positivist research, including the unit of analysis method [30,38,104-106] and the notion that external validity is a desirable research outcome $[15,29]$, both employed and espoused, respectively, in this paper.

This paper looks beyond the "AR versus positivism" debate, and it does so for a reason. The reason is the argument made by Kock and Lau [65] that AR and positivism can hardly be placed in the same conceptual category. Arguably, AR is a research approach, like experimental research, not an epistemology, like positivism or interpretivism $[58,83,99]$. Thus, comparing AR with positivism is equivalent to comparing a "painting technique" (e.g., oil painting) with a "school of painting" (e.g., impressionism). That is, in the same way that oil painting cannot be directly compared with impressionism, AR cannot be directly compared with positivism. Thus AR cannot be opposed to positivism, even though it may not be the most appropriate research approach for traditional positivist inquiry [65]. Once this notion is more widely accepted by those who practice AR, the debate between them and those who subscribe to other research approaches that fall into the broad category of "positivist research" will be replaced by cooperation in the search for solutions to longstanding methodological challenges.

\section{Acknowledgements}

The author would like to thank the individuals and organizations that participated in the several iterations of his action research study for their time and support. Thanks are also due to the Editor and anonymous reviewers for their comments and suggestions.

\section{References}

[1] M. Ackerman, Augmenting the organizational memory: a field study of answer garden, in: R. Furuta, C. Neuwirth (Eds.), Proceedings of CSCW'94 Conference, The Asso- ciation for Computing Machinery, New York, NY, 1994, pp. $243-252$.

[2] M. Alavi, An assessment of electronic meeting systems in a corporate setting, Information \& Management 25 (4) (1993) $175-182$.

[3] C. Argyris, Integrating the Individual and the Organization, Wiley, New York, NY, 1964.

[4] C. Argyris, Double loop learning organizations, Harvard Business Review 55 (5) (1977) 115-125.

[5] C. Argyris, On Organizational Learning, Blackwell, Cambridge, MA, 1992.

[6] C. Argyris, R. Putnam, D.M. Smith, Action Science, JosseyBass, San Francisco, CA, 1985.

[7] H.J. Arnold, Moderator variables: a clarification of conceptual, analytic, and psycometric issues, Organization Behaviour and Human Performance 29 (4) (1982) 143-174.

[8] D. Avison, F. Lau, M.D. Myers, P. Nielson, Action research, Communications of the ACM 42 (1) (1999) 94-97.

[9] D. Avison, R. Baskerville, M.D. Myers, Controlling action research projects, Information Technology and People 14 (1) (2001) 28-45.

[10] R.P. Bagozzi, Causal Models in Marketing, Wiley, New York, NY, 1980.

[11] R.M. Baron, D.A. Kenny, The moderator-mediator variable distinction in social psychological research: conceptual, strategic, and statistical considerations, Journal of Personality and Social Psychology 51 (6) (1986) 1173-1182.

[12] R. Baskerville, Distinguishing action research from participative case studies, Journal of Systems and Information Technology 1 (1) (1997) 25-45.

[13] R. Baskerville, Investigating information systems with action research, Communications of the Association for Information Systems 2 (Article 19) (1999). Available at: http:// cais.isworld.org.

[14] H.S. Becker, Theory: the necessary evil, in: D.J. Flinders, G.E. Mills (Eds.), Theory and Concepts in Qualitative Research: Perspectives from the Field, Teachers College Press, Columbia University, New York, 1993, pp. 218-229.

[15] L. Berkowitz, E. Donnerstein, External validity is more than skin deep: some answers to criticisms of laboratory experiments, American Psychologist 37 (3) (1982) 245-257.

[16] E. Brynjolfsson, L. Hitt, Is information systems spending productive? New evidence and new results, in: J.I. Degross, R.P. Bostrom, D. Robey (Eds.), Proceedings of the 14th International Conference on Information Systems, The Association for Computing Machinery, New York, NY, 1993, pp. $47-64$.

[17] C. Bunning, Placing Action Learning and Action Research in Context, International Management Centre, Brisbane, Australia, 1995.

[18] G. Burke, J. Peppard, Business process re-engineering: research directions, in: G. Burke, J. Peppard (Eds.), Examining Business Process Re-engineering, Kogan Page, London, 1995 , pp. $25-37$.

[19] D.B. Candlin, S. Wright, Managing the introduction of expert systems, International Journal of Operations and Production Management 12 (1) (1991) 46-59. 
[20] E.G. Carmines, R.A. Zeller, Reliability and Validity Assessment, Sage, Beverly Hills, CA, 1979.

[21] D. Cartwright (Ed.), Field Theory in Social Science, Harper \& Row, New York, NY, 1951.

[22] J.I. Cash Jr., P.R. Lawrence (Eds.), The Information Systems Research Challenge: Qualitative Research Methods, Harvard Business School, Boston, MA, 1989.

[23] J. Champy, Reengineering Management, Harper Business, New York, NY, 1995.

[24] P. Checkland, Systems Thinking, Systems Practice, Wiley, New York, NY, 1981.

[25] P. Checkland, From framework through experience to learning: the essential nature of action research, in: H. Nissen, H.K. Klein, R. Hirschheim (Eds.), Information Systems Research: Contemporary Approaches and Emergent Traditions, North-Holland, New York, NY, 1991, pp. 397-403.

[26] L. Chidambaram, B. Jones, Impact of communication medium and computer support on group perceptions and performance: a comparison of face-to-face and dispersed meetings, MIS Quarterly 17 (4) (1993) 465-491.

[27] T.Y. Choi, O.C. Behling, Top managers and TQM success: one more look after all these years, The Academy of Management Executive 11 (1) (1997) 37-47.

[28] A. Clement, Computing at work: empowering action by low-level users, Communications of ACM 37 (1) (1994) $53-63$.

[29] T.D. Cook, D.T. Campbell, Four kinds of validity, in: M.D. Dunnette (Ed.), Handbook of Industrial and Organizational Psychology, Rand McNally, Chicago, IL, 1976, pp. 224-246.

[30] J.W. Creswell, Research Design: Qualitative and Quantitative Approaches, Sage, Thousand Oaks, CA, 1994.

[31] R.L. Daft, R.H. Lengel, Organizational information requirements, media richness and structural design, Management Science 32 (5) (1986) 554-571.

[32] T.H. Davenport, Process Innovation, Harvard Business Press, Boston, MA, 1993.

[33] J.A. Davis, The Logic of Causal Order, Sage, London, England, 1985.

[34] R. Davison, GSS and action research in the Hong Kong police, Information Technology and People 14 (1) (2001) $60-78$.

[35] A.R. Dennis, R.M. Daniels Jr., G. Hayes, J.F. Nunamaker Jr., Methodology-driven use of automated support in business process re-engineering, Journal of Management Information Systems 10 (3) (1993) 117-138.

[36] A.R. Dennis, G.S. Hayes, R.M. Daniels Jr., Business process modeling with group support systems, Journal of Management Information Systems 15 (4) (1999) 115-142.

[37] T. Dobzhansky, Mankind Evolving: The Evolution of the Human Species, Yale Univ. Press, New Haven, CT, 1971.

[38] C.J. Drew, M.L. Hardman, Designing and Conducting Behavioral Research, Pergamon, New York, NY, 1985.

[39] M. Elden, R.F. Chisholm, Emerging varieties of action research, Human Relations 46 (2) (1993) 121-141.

[40] J. Forman, J. Rymer, The genre system of the Harvard case method, Journal of Business and Technical Communication 13 (4) (1999) 373-400.
[41] W.M. Fox, An interview with Eric Trist, father of the sociotechnical systems approach, The Journal of Applied Behavioural Science 26 (2) (1990) 259-279.

[42] D. Francis, Moving from non-interventionist research to participatory action, in: C. Collins, P. Chippendale (Eds.), Proceedings of The First World Congress on Action Research, vol. 2, Acorn, Sunnybank Hills, Queensland, Australia, 1991.

[43] J. Fulk, J. Schmitz, C.W. Steinfield, A social influence model of technology use, in: J. Fulk, C. Steinfield (Eds.), Organizations and Communication Technology, Sage, Newbury Park, CA, 1990, pp. 117-140.

[44] R.D. Galliers, In search of a paradigm for information systems research, in: E. Mumford (Ed.), Research Methods in Information Systems, North-Holland, New York, NY, 1984, pp. $281-297$.

[45] R.D. Galliers, Choosing appropriate information system research approaches: a revised taxonomy, in: H. Nissen, H.K. Klein, R. Hirschheim (Eds.), Information Systems Research: Contemporary Approaches and Emergent Traditions, NorthHolland, New York, NY, 1991, pp. 327-345.

[46] R.D. Galliers, Choosing information systems research approaches, in: R. Galliers (Ed.), Information Systems Research: Issues, Methods and Practical Guidelines, Blackwell, Boston, MA, 1992, pp. 144-162.

[47] R.D. Galliers (Ed.), Information Systems Research: Issues, Methods and Practical Guidelines, Blackwell, Boston, MA, 1992

[48] R.D. Galliers, A manifesto for information management research, British Journal of Management 6 (1995, Dec.) 45-52.

[49] R.B. Gallupe, W.H. Cooper, M. Grise, L.M. Bastianutti, Blocking electronic brainstorms, Journal of Applied Psychology 79 (1) (1994) 77-86.

[50] D.A. Gioia, H.P. Sims Jr., Social cognition in organizations, in: H.P. Sims Jr., D.A. Gioia (Eds.), The Thinking Organization, Jossey-Bass, San Francisco, CA, 1986.

[51] B.G. Glaser, Theoretical Sensitivity: Advances in the Methodology of Grounded Theory, Sociology Press, Mill Valley, CA, 1978.

[52] B.G. Glaser, Emergency Versus Forcing: Basics of Grounded Theory Analysis, Sociology Press, Mill Valley, CA, 1992.

[53] B.G. Glaser, A.L. Strauss, The Discovery of Grounded Theory: Strategies for Qualitative Research, Aldine Publishing, Chicago, IL, 1967.

[54] D. Gregory, H. Ward, Statistics for Business Studies, McGraw-Hill, London, England, 1974.

[55] B. Gustavsen, Action research and the generation of knowledge, Human Relations 46 (11) (1993) 1361-1365.

[56] M. Hammer, J. Champy, Reengineering the Corporation, Harper Business, New York, NY, 1993.

[57] F. Heller, Another look at action research, Human Relations 46 (10) (1993) 1235-1242.

[58] R.A. Hirschheim, Information systems epistemology: a historical perspective, in: E. Mumford (Ed.), Research Methods in Information Systems, North-Holland, New York, NY, 1985, pp. 13-35.

[59] S. Jonsonn, Action research, in: H. Nissen, H.K. Klein, R. Hirschheim (Eds.), Information Systems Research: Contem- 
porary Approaches and Emergent Traditions, North-Holland, New York, NY, 1991, pp. 371-396.

[60] J.I. Karlsen, Action research as a method, in: W.F. Whyte (Ed.), Participatory Action Research, Sage, Newbury Park, CA, 1991, pp. 143-158.

[61] L.D. Ketchum, E. Trist, All Teams are not Created Equal, Sage, Newbury Park, CA, 1992.

[62] N. Kock, Negotiating mutually satisfying IS action research topics with organizations: an analysis of Rapoport's initiative dilemma, Journal of Workplace Learning 9 (7) (1997) 253-262.

[63] N. Kock, Process Improvement and Organizational Learning: The Role of Collaboration Technologies, Idea Group Publishing, Hershey, PA, 1999.

[64] N. Kock, Asynchronous and distributed process improvement: the role of collaborative technologies, Information Systems Journal 11 (2) (2001) 87-110.

[65] N. Kock, F. Lau, Information systems action research: serving two demanding masters, Information Technology and People 14 (1) (2001) 6-12.

[66] N. Kock, D. Avison, R. Baskerville, M. Myers, T. WoodHarper, IS action research: can we serve two masters? in: P. De, J. DeGross (Eds.), Proceedings of the 20th International Conference on Information Systems, The Association for Computing Machinery, New York, NY, 1999, pp. $582-585$.

[67] N. Kock, R.J. McQueen, J.L. Scott, Can action research be made more rigorous in a positivist sense? The contribution of an iterative approach, Journal of Systems and Information Technology 1 (1) (1997) 1-24.

[68] M.C. Lacity, M.A. Janson, Understanding qualitative data: a framework of text analysis methods, Journal of Management Information Systems 11 (2) (1994) 137-155.

[69] F. Lau, A review on the use of action research in information systems studies, in: A.S. Lee, J. Liebenau, J.I. DeGross (Eds.), Information Systems and Qualitative Research, Chapman \& Hall, London, England, 1997, pp. 31-68.

[70] G.E. Ledford, S.A. Mohrman, Self-design for high involvement: a large-scale organizational change, Human Relations 46 (2) (1993) 143-173.

[71] G.E. Ledford, S.A. Mohrman, Looking backward and forward at action research, Human Relations 46 (11) (1993) 1349-1359.

[72] K. Lewin, Action research and minority problems, in: G.W. Lewin (Ed.), Resolving Social Conflicts, Harper \& Row, New York, 1946, pp. 201-216.

[73] G.W. Lewin (Ed.), Resolving Social Conflicts, Harper \& Row, New York, NY, 1948.

[74] A. Majchrzak, R.E. Rice, A. Malhotra, N. King, S. Ba, Technology adaptation: the case of a computer-supported interorganizational virtual team, MIS Quarterly 24 (4) (2000) $569-600$.

[75] M.L. Markus, Electronic mail as the medium of managerial choice, Organization Science 5 (4) (1994) 502-527.

[76] J. McKay, P. Marshall, The dual imperatives of action research, Information Technology and People 14 (1) (2001) 46-60.
[77] R. McTaggart, Principles for participatory action research, Adult Education Quarterly 41 (3) (1991) 168-187.

[78] M.B. Miles, A.M. Huberman, Qualitative Data Analysis: An Expanded Sourcebook, Sage, London, England, 1994.

[79] E. Mumford, Advice for an action researcher, Information Technology and People 14 (1) (2001) 12-27.

[80] R.E. Neilson, Collaborative Technologies and Organizational Learning, Idea Group Publishing, Hershey, PA, 1997.

[81] W.J. Orlikowski, Learning from notes: organizational issues in groupware implementation, in: J. Turner, R. Kraut (Eds.), Proceedings of CSCW'92 Conference, The Association for Computing Machinery, New York, NY, 1992, pp. $362-369$.

[82] W.J. Orlikowski, Learning from notes: organizational issues in groupware implementation, Information Society 9 (3) (1993) $237-251$.

[83] W.J. Orlikowski, J.J. Baroudi, Studying information technology in organizations: research approaches and assumptions, Information Systems Research 2 (1) (1991) 1-28.

[84] G.P. Pervan, D.J. Klass, The use and misuse of statistical methods in information systems research, in: R. Galliers (Ed.), Information Systems Research: Issues, Methods and Practical Guidelines, Blackwell, Boston, MA, 1992, pp. $208-229$.

[85] M. Peters, V. Robinson, The origins and status of action research, The Journal of Applied Behavioral Science 20 (2) (1984) 113-124.

[86] E.M. Phillips, D.S. Pugh, How to Get a PhD: A Handbook for Students and Their Supervisors, Open Univ. Press, Bristol, PA, 2000.

[87] R.N. Rapoport, Three dilemmas in action research, Human Relations 23 (6) (1970) 499-513.

[88] P. Reason (Ed.), Human Inquiry in Action, Sage, Newbury Park, CA, 1988.

[89] P. Reason, Sitting between appreciation and disappointment: a critique of the special edition of human relations on action research, Human Relations 46 (10) (1993) 1253-1270.

[90] W.M. Riggs, W.H. Bellinger, D.B. Krieger, The impact of groupware: work process automation and organizational learning, Technology Analysis \& Strategic Management 8 (3) (1996) 271-283.

[91] D.L. Schacter, The Seven Sins of Memory: How the Mind Forgets and Remembers, Houghton Mifflin, New York, NY, 2001.

[92] J. Short, E. Williams, B. Christie, The Social Psychology of Telecommunications, Wiley, London, England, 1976.

[93] R. Sommer, Serving two masters, The Journal of Consumer Affairs 28 (1) (1994) 170-187.

[94] B. Sommer, R. Sommer, A Practical Guide to Behavioral Research, Oxford Univ. Press, New York, NY, 1991.

[95] A.L. Strauss, J.M. Corbin, Basics of Qualitative Research: Grounded Theory Procedures and Techniques, Sage, Newbury Park, CA, 1990.

[96] A.L. Strauss, J.M. Corbin, Basics of Qualitative Research: Techniques and Procedures for Developing Grounded Theory, Sage, Newbury Park, CA, 1998.

[97] G.I. Susman, R.D. Evered, An assessment of the scientific 
merits of action research, Administrative Science Quarterly 23 (4) (1978) 582-603.

[98] B.C.Y. Tan, K. Wei, W.W. Huang, G. Ng, A dialogue technique to enhance electronic communication in virtual teams, IEEE Transactions on Professional Communication 43 (2) (2000) 153-165.

[99] J. Teichman, K.C. Evans, Philosophy: A Beginner's Guide, Blackwell, Oxford, UK, 1995.

[100] E.M. Trauth, B. O'Connor, A study of the interaction between information technology and society: an illustration of combined qualitative research methods, in: H. Nissen, H.K. Klein, R. Hirschheim (Eds.), Information Systems Research: Contemporary Approaches and Emergent Traditions, NorthHolland, New York, NY, 1991, pp. 131-143.

[101] D.P. Truex III, Three issues concerning relevance in IS research: epistemology, audience, and method, Communications of the AIS 6 (Article 24) (2001). Available at: http:// cais.isworld.org.

[102] S.J. Winter, The symbolic potential of computer technology: differences among white-collar workers, in: J.I. Degross, R.P. Bostrom, D. Robey (Eds.), Proceedings of 14th International Conference on Information Systems, The Association for Computing Machinery, New York, 1993, pp. 331-344.

[103] J.C. Woofford, Getting inside the leader's head: a cognitive processes approach to leadership, SAM Advanced Management Journal 59 (3) (1994) 4-9.
[104] R.K. Yin, The case study crisis: some answers, Administrative Science Quarterly 26 (1981, March) 58-65.

[105] R.K. Yin, Research design issues in using the case study method to study management information systems, in: J.I. Cash, P.R. Lawrence (Eds.), The Information Systems Research Challenge: Qualitative Research Methods, Harvard Business School, Boston, MA, 1989, pp. 1-6.

[106] R.K. Yin, Case Study Research, Sage, Newbury Park, CA, 1994.

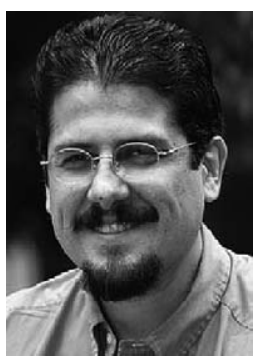

Ned Kock is the Class of the 1961 Associate Professor and Director of the E-Collaboration Research Center, in the College of Business and Economics, at Lehigh University. He holds a $\mathrm{PhD}$ in information systems from the University of Waikato, New Zealand. He is the author/co-author of four books, including Process Improvement and Organizational Learning: The Role of Collaboration Technologies. Ned's research interests are focused on electronic communication tools and their effects on groups performing complex, knowledgeintensive tasks. He is currently developing a theoretical framework that tries to explain behavior toward electronic communication technologies based on a Darwinian evolutionary perspective. 


\section{Decision Support Systems}

\section{Editor-in-Chief}

Andrew B. Whinston

MSIS Department CBA 5-202

University of Texas-Austin

Austin, TX 78712

USA

Tel.: (512) 471-8879

Fax: (512) 471-0587

\section{Area Editors}

1. DSS Foundations
CLYDE W. HoLSAPPLE
Dept. of Decision Science and
Information Systems
Business and Economics Building
University of Kentucky
Lexington, KY $40506-0034$
USA
2. DSS Development-Functionality
ROBERT W. BLANNING
Owen Graduate School of Management
401 21st Ave South
Vanderbilt University
Nashville, TN 37203
USA
3. DSS Development-Interfaces
T.P. LIANG
College of Management
National Sun Yat-sen University
Kaohsiung, Taiwan 80424
R.O.C.
4. DSS Impacts and Evaluation
JAMES MARSDEN
School of Business Administration
University of Connecticut
368 Fairfield Rd., Rm. 302, U-41IM
Storrs, CT 06269
USA
5. DSS Reference Studies
VIJAY S. MoOKERJEE
Dept. of Management
School of Business
Univ. of Texas at Dallas
Richardson, TX 75083-0688
USA
6. DSS Experiences, Management
and Education
H.G. SoL
Delft University of Technology
Faculty of Technology, Policy and
Management
Jaffalaan 5
2628 BX Delft
THE NETHERLANDS
G. KoEHLER
School of Business Admin
Decision and Information Sciences
University of Florida
Gainesville, FL 32611
USA
7. DSS Digital and Web Computing
HsinCHUN CHEN
MIS Department
MCCL $430 Z$
Tucson, AZ 85721
USA

\section{Associate Editors}

R. AgARWAL

University of Maryland

College of Business and Management

IMS Department

College Park, MD 20742

USA

S. BA

Dept. of Operations and
Information Management

Information Managem
School of Business

School of Business
Univ. of Connecticut

2100 Hollside Road

Storres, CT 06269

USA

ANDREW D. BAILEY, JR.

College of Business

The University of Arizona

Tucson, AZ 85721

USA

KURT BAUKNECHT

Universität Zürich

Winterthurerstrasse 190

$\mathrm{CH}-8057$ Zürich

SWITZERLAND

HEMANT K. BHARGAVA

Graduate School of

AOB IV, Room 135

One Shields Avenue

Univ. of California Davis

Davis, CA 95616

USA

\section{Editorial Assistant \\ Veronika Whinston \\ MSIS Department CBA 5-202 \\ University of Texas-Austin \\ Austin, TX 78712 \\ Tel.: (512) 471-8879 \\ Fax: (512) 471-0587 \\ Email: dss@uts.cc.utexas.edu}

$\begin{array}{ll}\begin{array}{l}\text { FRANÇOIS BODART } \\ \text { Université Notre Dame de la Paix }\end{array} & \begin{array}{l}\text { VARGHESE S. JACOB } \\ \text { The University of Texas at Dallas } \\ \text { Rue Grandgagnage 21 }\end{array} \\ \text { School of Management } \\ \text { B-500 Namur } & \text { Management Science \& Information } \\ \text { BELGIUM } & \text { Systems } \\ \text { GIAMPIO BRACCHI } & \text { 2601 N. Floyd Road, J044/RM. } \\ \text { Politecnico di Milano } & \text { TX 75083-0688, } \\ \text { Piazza Leonardo da Vinci 32 } & \text { USA } \\ \text { I-20-133 Milano } & \text { WoLFGANG JANKO } \\ \text { ITALY } & \text { Wirtschaftsuniversität } \\ \text { F.V. BURSTEIN } & \text { Auegasse 2-6 } \\ \text { School of Information Management } & \text { A-1096 Wien } \\ \text { and Systems } & \text { AUSTRIA } \\ \text { Faculty of Information Technology } & \text { Kou JisonG } \\ \text { Monash University } & \text { Institute of Information and Control (IIC) } \\ \text { P.O. Box 197 } & \text { Tianjin University } \\ \text { Melbourne, Vic. 3145 } & \text { Tianiin 300072 }\end{array}$

P.O. Box 197
Melbourne, Vic.

AUSTRALIA

JOHN BUTLER

The Ohio State University

2100 Neil Avenue

Columbus, $\mathrm{OH} 43210$

USA

JAMES I. CASH

Management Information Systems

Graduate School of Business

Harvard University

Anderson 34

Soldiers Field

USA

AI-MEI CHANG

Information Resources Management

College

National Defense University

Ft. McNair

Washington, DC 20319

USA

PRABUdDHA DE

Krannert School of Management

Purdue University

West Lafayette

USA

AMITAVA DUTTA

George Mason University

Decision Sciences and MIS

School of Business Adm

Fairfax, VA 22030-4444
USA

A. GUPTA

University of Connecticut

368 Falirfeld Road U-41 IM

Storrs, CT 06269-2041

USA

CHRIstopher HABEL

Universität Hamburg

Fachbereich Informatik

Vogt-Köln-Str 30

D-22527 Hamburg

GERMANY

TERRENCE HENDERSHOTT

Haas School of Business

Univ. of California at Berkeley

Berkeley, CA 94720-1900

USA

NORBERT HENRICHS

Universität Düsseldorf

Philosophisches Institut

D-40225 Düsseldorf

GERMANY

A. HEVNER

University of South Florid

College of Business

4202 E. Fowler Ave. CIS1040

Tampa, FL 33620

USA

WEN-LING HSU

AT \& T Labs.

$1 \mathrm{CO} 3$ Building 104

180 Park Ave.

Florham Park, NJ 09732

USA

PATRICK HUMPHREYS

London School of Economics

Houghton Street

London WC2A 2AE

UK

Carnegie Mellon University
D. LEIDNER

Information Systems Dept.

Baylor University

Waco, TX 7698-8005

USA

JEAN LOUIS LE MOIGNE

Université d'Aux-Marseille III

5 Avenue Victor Hugo

13100 Aix-en-Provence

FRANCE

MATS LUNDEBERG

Stockholm School of Economics

Handelshögskolan

I Stockholm

Saltmätargatan 13-17

P.O. Box 6501

11383 Stockholm

SWEDEN

M. MANNINO

Management Information System

Graduate School of Business

University of Colorado

P.O. Box 173364

Denver, CO 80217-3364

USA

Peter Mertens

Universität Erlangen Nürnberg

Portfach 3931

D-90020 Nürnberg

GERMANY

LEIF B. METHLIE

Norges Handelshogskole

Helleveien 30

5000 Bergen-Sandviken

Tianjin 300072

P.R. OF CHINA

P.K. KANNAN

NORWAY

BERND E. MEYER

The Robert H. Smith School of Business Hermann-Münzing Str. 15

University of Maryland

USA

MELODY Y. KIANG

Information Systems Department

College of Business Administration

California State University at Long Beach

1250 Bellflower Blvd.

Long Beach, CA 90840

USA

MICHEL KLEIN

Centre d'Enseignement Supérieur

des Affaires

1 Rue de la Liberation

78350 Jouy-en-Josas

FRANCE

HIROSHI KONNO

Tokyo Institute of Technology

2-12-1 Oh-Okayama, Meguru-k

Tokyo 152

JAPAN

BENN R. KONSYNSKI

Emory University

School of Business

Decision of Information Analysis

Atlanta, GA 30322

USA

Hermann Krallmann

Technische Universität Berlin

Franklinstrasse 28/29

D-10587 Berlin

GERMANY

RAMAYYA KRISHNAN

H. John Heinz III School of

Public Policy and Management

Pittsburgh, PA 15214

USA

HSIANGCHU LAI

Department of Information Management

National Sun Yat-sen University

Hsi-Tze Wan, Kaohsiung 80424

Taiwan
R.O.C.

FRANK LAND

Stowford Barton

Harford, Ivy Bridge

Devon, PL21 OJD

KARL R. LANG

City Univ of New York

Dept. of Computer Information Systems

Zicklin School of Business

Baruch College

17 Lexington Avenue,

USA

JAE KYu LeE

Department of Management Science

Korea Advanced Institute of Science

and Technology

and Technology

P.O. Box
Seoul
KOREA

RONALD M. LEE

Erasmus University Research Centre

for Decision and Information Systems

Erasmus University Rotterdam

Burg. Oudlaan 50

3062 PA Rotterdan

THE NETHERLANDS

RADHIKAR SANTHANAM

DSIS Area

Gatton College of Business \&

Economics

Univ. of Kentucky

Lexington, KY 40506-0034

USA

FABIO A. SchreIBER

Politecnico di Milano

Piazza Leonardo da Vinci, 32

20133 Milano

ITALY

A. SEGEV

University of California

Hass School of Busines

Berkeley, CA 94720-1900

AMILCAR SERnAdAs

NES

Rua Alves Redol 9, 2

1000 Lisboa

PORTUGAL

PROFESSOR RAMESH SHARDA

Dept. of Management Science

and Info. Sys.

Oklahoma State Univ.

Stillwater, OK 74078

USA

MICHAEL JENG-PING SHAW

Department of Business Administration

University of Illinois

Commerce Buildin

1206 S. Sixth St.

Champaign, IL 61820-627

USA

PROFESSOR MICHAEL SMITH

H. John Heinz III

Carnegie Mellon Univ.

4800 Forbes Avenue, HBH 2105D

Pittsburgh, PA 15213

USA

Tel: 412-268-5978

E-mail: mds@cmu.edu

EDWARD A. STOHR

Center for Research and Information

Stern School of Business

New York University

44 West 4th Street

New York, NY 10012-1126

USA

V.C. STOREY

Georgia State University

CIS, Department of Business

Administration

Atlanta, GA 30302-4015

USA 